\title{
Qualidade da madeira de Eucalyptus benthamii para produção de celulose por espectroscopia no infravermelho próximo (NIRS)
}

\author{
Quality of Eucalyptus benthamii wood for pulp production by Near Infrared \\ Spectroscopy (NIRS)
}

Talita Baldin ${ }^{1}$ (D), Maiara Talgatti² (1), Amanda Grassmann da Silveira² (D), José Newton Cardoso Marchiori ${ }^{2}$ (D), Gleison Augusto dos Santos ${ }^{3}$ (D), Osmarino Pires dos Santos ${ }^{4}$ (iD, Brígida Maria dos Reis Teixeira Valente ${ }^{4}$ (i)

${ }^{1}$ Universidade Federal de Minas Gerais - UFMG, Montes Claros, MG, Brasil

${ }^{2}$ Universidade Federal de Santa Maria - UFSM, Santa Maria, RS, Brasil

3Universidade Federal de Viçosa - UFV, Viçosa, MG, Brasil

${ }^{4} \mathrm{CMPC}$ Celulose Riograndense, Guaíba, RS, Brasil

Como citar: Baldin, T., Talgatti, M., Silveira, A. G., Marchiori, J. N. C., Santos, G. A., Santos, O. P., \& Valente, B. M. R. T. (2020). Qualidade da madeira de Eucalyptus benthamii para produção de celulose por espectroscopia no infravermelho próximo (NIRS). Scientia Forestalis, 48(126), e3192. https://doi.org/10.18671/scifor.v48n126.05

\begin{abstract}
Resumo
O uso de práticas não destrutivas como a espectroscopia na região do infravermelho próximo (Near Infrared Spectroscopy, NIRS) tem crescido e se destacado em qualificações de processos e produtos, e também em programas de melhoramento genético florestal, pois permitem a seleção de árvores ainda no povoamento. O objetivo deste estudo foi utilizar o NIR como ferramenta na análise da qualidade da madeira e seleção genética de árvores de Eucalyptus benthamii Maiden \& Cambage com vistas à produção de celulose. Para o desenvolvimento dos modelos de calibração, 87 árvores, sendo 75 de E. benthamii, quatro de $E$. dunnii, quatro de E. grandis e quatro de E. saligna foram transformadas em cavacos. Parte desse material serviu para as análises de lignina Klason e totais, holocelulose e densidade básica, outra parte foi moída para aquisição dos espectros, que posteriormente foram correlacionados aos valores laboratoriais. A calibração do modelo foi determinada por análise de regressão dos mínimos quadrados parciais (PLS). Os modelos de calibração apresentaram ajustes satisfatórios para a lignina Klason $\left(R^{2}=0,82\right)$, lignina total $\left(R^{2}=0,74\right)$, teor de holocelulose $\left(R^{2}=0,82\right)$ e densidade básica de madeira $\left(R^{2}=0,73\right)$ e a sua utilização comercial é recomendada para predição de amostras desconhecidas. A técnica NIRS se mostrou promissora na predição da qualidade da madeira de Eucalyptus benthamii para a indústria de celulose e pode ser utilizada para acelerar o programa de melhoramento genético do $E$. benthamii.
\end{abstract}

Palavras-chave: Espectroscopia no infravermelho próximo; Avaliação não destrutiva; Modelos de calibração; Qualidade da madeira; Química da madeira.

\begin{abstract}
The use of non-destructive techniques such as near infrared spectroscopy (NIRS) has become of great interest in characterization of wood quality. Additionally, non-destructive techniques offer great application in genetic improvement programs because they aid selection of trees that are still in the stands. The objective of this study was to assess the suitability of NIRS as a tool in the analysis of wood quality and genetic selection of Eucalyptus benthamii Maiden \& Cambage clones used for production of cellulose. Eighty-seven trees ( 75 of E. benthamii, 4 of E. dunnii, 4 of E. grandis, and 4 of E. saligna) were used for the development of calibration models. Wood samples were analyzed for Klason lignin, total lignin, holocellulose, and basic density. A sub-sample was milled and used to acquire the spectra, which were
\end{abstract}

Fonte de financiamento: Coordenação de Aperfeiçoamento de Pessoal de Nível Superior - CAPES; CMPC Celulose Riograndense. Número de processo não se aplica.

Conflito de interesse: Nada a declarar.

Autor correspondente: talita.baldin@hotmail.com

Recebido: 21 novembro 2018.

Aceito: 2 julho 2019.

Editor: Francides Gomes Silva Júnior

(c) (†) Este é um artigo publicado em acesso aberto (Open Access) sob a licença Creative Commons Attribution, que permite uso, distribuição e reprodução em qualquer meio, sem restrições desde que o trabalho original seja corretamente citado. 
then compared against values obtained from standard laboratory techniques. Model calibration was determined by partial least squares (PLS) regression analysis. The values observed for anatomical and chemical traits of Eucalyptus benthamii wood remained within the range considered appropriate for Klason lignin $(R 2=0.82)$ and total lignin content $(R 2=0.74)$. NIRS calibration models presented satisfactory adjustments for holocellulose content $(R 2=0.82)$ and wood density $(R 2=0.73)$. The NIRS models developed in this study offer the cellulose industry a viable commercial tool that can be used for characterization of samples of Eucalyptus benthamii wood. The accuracy of the models warrants their use in programs to accelerate the genetic improvement of $E$. benthamii.

Keywords: Near infrared spectroscopy; Non-destructible technique; Prediction models; Wood quality; Wood chemistry.

\section{INTRODUÇÃO}

Nos programas de melhoramento genético brasileiros voltados a produção de celulose e papel, os principais fatores considerados para a seleção das espécies envolveram, por muitas décadas, a taxa de crescimento das árvores, representada pelo incremento médio anual (Gomide et al., 2005); a densidade básica da madeira (Raymond \& Muneri, 2001); e, mais recentemente o incremento médio anual de celulose na floresta (ton. ha ${ }^{-1}$ ano $^{-1}$ ), representado pelo IMACel (Segura, 2015).

O sucesso silvicultural obtido é expressivo e está refletido nos dados que apontam o país como o segundo produtor mundial de celulose de todos os tipos e o oitavo produtor de papel, alcançando 18 e 10 milhões de toneladas, respectivamente (Indústria Brasileira de Árvores, 2017).

Sabe-se, contudo, que outras características também devem ser adicionalmente avaliadas na seleção de espécies para produção de celulose, como a qualidade tecnológica da madeira, definida dentre outras propriedades, pela sua composição química e anatômica, bem como o processo e a condição de formação da polpa celulósica durante o cozimento (Segura et al., 2017). Atualmente, o termo qualidade da madeira tem assumido um aspecto fundamental na área de celulose, pois possibilita a geração de produtos de alta qualidade para diferentes segmentos de mercado.

Um diagnóstico fiável da qualidade da madeira geralmente requer muito tempo (dias ou meses) para ser determinado em laboratório, além de ser um processo normalmente caro, aplicável em um número restrito de amostras e, na maioria das vezes utiliza métodos destrutivos. No entanto, para a caracterização de seus produtos, as empresas que trabalham com a produção florestal demandam métodos rápidos e precisos, que possam ser aplicados em um grande número de amostras obtidas, preferencialmente, de árvores vivas do povoamento, para que as propriedades da madeira possam ser mapeadas e melhoradas geneticamente, visando seu melhor aproveitamento e a redução dos custos.

Nesse cenário, é crescente a adoção de metodologias de avaliação não destrutivas, que refletem em reduzidos custos e tempo de análises da madeira. A espectroscopia no infravermelho próximo (Near Infrared Spectroscopy, NIRS) tem se mostrado como uma ferramenta eficiente no controle de qualidade industrial. NIRS é uma técnica rápida, acurada e aplicável a um grande número de amostras (Muniz et al., 2012). Adicionalmente não requer o uso de reagentes químicos, é parcialmente independente de erros de operador e utiliza amostras com reduzida preparação prévia.

$\mathrm{Na}$ análise da qualidade da madeira o NIRS surge como um método que pode ser considerado não invasivo, capaz de predizer suas propriedades químicas (Pecoraro et al., 2015), anatômicas (Hein et al., 2012), físicas (Rosso et al., 2013) e mecânicas (Kelley et al., 2004). A tecnologia também é aplicada na verificação da qualidade da polpa celulósica na indústria, com a determinação, por exemplo, da relação lignina Siringil/Guaiacil (Ramadevi et al., 2016) e dos teores de holocelulose e lignina total (Zhou et al., 2015, 2016). Esse tipo de análise é de grande valia para os programas de melhoramento genético, visto que muitas vezes não é possível abater a árvore que é um material genético único e de valor para o avanço das gerações (Estopa, 2017). 
Diante do exposto, objetiva-se utilizar a técnica de espectroscopia no infravermelho próximo como ferramenta na análise da qualidade da madeira e seleção genética de árvores de Eucalyptus benthamii Maiden \& Cambage para produção de celulose.

\section{MATERIAL E MÉTODOS}

\section{Caracterização geral do experimento}

A madeira de Eucalyptus benthamii foi proveniente de um plantio experimental com quatro anos de idade de propriedade da empresa CMPC - Celulose Riograndense, localizado no município de Encruzilhada do Sul, Rio Grande do Sul, Brasil, sob as coordenadas $30^{\circ} 55^{\prime} 26,8^{\prime \prime}$ S, 52 46'83,9" W. O povoamento foi implantado no ano de 2011, em uma área com 1,3 hectares, com as espécies de Eucalyptus benthamii, E. dunnii, E. grandis e E. saligna aleatoriamente plantadas na área.

\section{Preparação das amostras para construção dos modelos de calibração}

Para a seleção das amostras que constituíram o modelo de calibração NIRS, inicialmente retirou-se uma alíquota de serragem da madeira de 1.219 árvores no povoamento (número de árvores vivas e em condições de amostragem). Destas, 1.190 eram de Eucalyptus benthamii, 14,6 e 9 de $E$. dunnii, E. grandis e E. saligna, respectivamente. Estas últimas espécies também foram amostradas para compor o modelo de calibração, tornando-o mais robusto e capaz de predizer novas amostras.

Para a coleta da serragem foi utilizado o método não destrutivo: com o auxílio de uma furadeira e uma broca a serragem foi coletada em quatro pontos distintos na altura do DAP (diâmetro a altura do peito), homogeneizada e acondicionada em sacos de papel. Posteriormente foi processada em partículas menores em moinho de facas e a fração utilizada constou daquela que atravessou a peneira com furos de $1 \mathrm{~mm}$ (16 mesh). A serragem foi então enviada para o laboratório de Espectroscopia de Infravermelho do Instituto Superior de Agronomia, Lisboa, Portugal. No laboratório foram coletados espectros das 1.219 amostras, sem preparação prévia, utilizando espectrômetro de infravermelho próximo em transformada de Fourier (FT-NIR), modelo MPA, da Bruker.

A partir dos espectros, foram estimados os parâmetros de rendimento total em celulose e a relação da lignina Siringil/Guaiacil (S/G) de todas as árvores do povoamento, fazendo uso de modelos de calibração de Eucalyptus globulus e E. grandis, desenvolvidos em estudos anteriores no laboratório de Espectroscopia no Infravermelho (Alves et al., 2011, 2012). Os valores estimados foram utilizados para selecionar 20 árvores com alto rendimento em celulose (55 até 62\%) e 20 com baixo (36 até 43\%) e outras 37 com valores intermediários (44 até 54\%). Adicionalmente, cinco árvores foram selecionadas para representar altos valores S/G $(1,70$ até 2,10$)$ e mais cinco com baixos valores de $S / G(1,10$ até 1,69). No total, 87 árvores, sendo 75 de E. benthamii, 4 de E. dunnii, 4 de E. grandis e 4 de E. saligna, foram escolhidas para serem novamente amostradas. A seleção das 12 amostras de outros eucaliptos teve por finalidade proporcionar a confecção de um modelo de calibração heterogêneo e robusto, envolvendo a maior variabilidade de dados possível.

Após a seleção das 87 novas árvores, coletou-se um torete de cada uma, com tamanho de aproximadamente $70 \mathrm{~cm}$, o mais próximo possível do DAP e posteriormente picados em cavacos. Parte desse material foi enviado ao Centro I+D da CMPC - Celulose S.A., na planta de Santa Fé, Chile, para os ensaios convencionais de química úmida e outra parte ao laboratório de produtos florestais, LPF - Universidade Federal de Santa Maria, onde foi moído nas mesmas condições anteriores (moinho de facas, dotado de peneira com orifícios de $1 \mathrm{~mm}$ ) e enviado para aquisição dos espectros no laboratório de Espectroscopia de Infravermelho da Universidade em Portugal. 


\section{Caracterização da madeira em laboratório}

As propriedades químicas de teor de lignina Klason (Technical Association of the Pulp and Paper Industry, 2002), lignina total (Technical Association of the Pulp and Paper Industry, 1991) e holocelulose (Technical Association of the Pulp and Paper Industry, 1999) e a densidade básica (Scandinavian Pulp, Paper and Board Testing Committee, 1995) foram convencionalmente determinados para as 87 amostras de madeira e constituíram o conjunto das variáveis dependentes para as quais foram ajustados os modelos de calibração.

\section{Desenvolvimento e seleção dos modelos de calibração}

Os espectros das 87 amostras foram associados com os valores das propriedades químicas das madeiras de Eucalyptus benthamii, E. dunnii, E. grandis e E. saligna determinados pelas técnicas padrão. O método de validação utilizado no estudo foi a validação-cruzada, porém, antes do processamento propriamente dito, os dados foram submetidos ao pré-processamento, sendo a primeira derivada $\left(1^{\text {st }}\right.$ Der); a normalização vetorial (VN); a primeira derivada $\left(1^{\text {st Der }) ~+~ a ~ n o r m a l i z a c ̧ a ̃ o ~ v e t o r i a l ~(V N) ~ e ~ a ~ p r i m e i r a ~}\right.$ derivada ( ${ }^{{ }^{\text {st}}}$ Der $)+$ a correção multiplicativa do sinal (MSC).

A calibração do modelo foi determinada por análise de regressão dos Mínimos Quadrados Parciais (PLS), conforme recomendação de Gierlinger et al. (2002). Os modelos foram ajustados com o número de variáveis latentes (VLs) necessárias para fornecer o melhor ajuste, sem perder a variância dos dados.

As faixas espectrais acima de $10.000 \mathrm{~cm}^{-1}$ foram excluídas, posto que, nessa região, o espectro apresenta repetições de ruídos que não tem informação relevante sobre a propriedade de interesse. Os valores definidos como outliers na elaboração do modelo pelo programa estatístico do software OPUS Quant 6.2 foram removidos.

A seleção dos modelos de calibração foi tomada com base no coeficiente de determinação $\left(R^{2}\right)$, na raiz quadrada do erro médio da validação cruzada (RMSECV) e na relação de desempenho do desvio (RPD) calculado como a razão entre o desvio-padrão dos valores de referência, e o erro padrão da validação cruzada. De acordo com Schimleck et al. (2003) deve possuir valor acima de 1,5 para o modelo ser considerado satisfatório na seleção de árvores em programas de melhoramento genético.

\section{Avaliação final do povoamento de Eucalyptus benthamii}

Os modelos de calibração desenvolvidos para as características químicas da madeira (teor de lignina Klason, lignina total e holocelulose) e para a densidade básica, serviram para as predições das 1.190 amostras de Eucalyptus benthamii do povoamento. Embora tenham sido utilizadas quatro amostras de E. dunnii, E. grandis e E. saligna para compor os modelos matemáticos, a predição das árvores restantes destas espécies no campo não é de interesse neste estudo.

Assim, os 1.190 espectros coletados da serragem obtida pelo método não destrutivo nas árvores vivas foram importados para o computador, e com auxílio do software OPUS Quant 6.2 todas as amostras tiveram valores estimados pelos modelos, conforme a propriedade de qualidade da madeira.

\section{RESULTADOS E DISCUSSÃO}

\section{Análise química e densidade básica da madeira}

A lignina teve sua quantidade total determinada pela soma das frações solúvel e insolúvel em ácido, também conhecida por lignina Klason. Os teores médios de lignina Klason e lignina total mostram-se menores em E. dunnii ( $23,6 \%$ e $27,8 \%$, respectivamente) e maiores em E. grandis (28\% e 31,6\%, respectivamente). Os resultados encontrados (Tabela 1) estão dentro da variação normal reportada em estudos químicos da madeira de Eucalyptus sp. (Zanuncio et al., 2013; Neiva et al., 2015; Souza et al., 2017; Estopa, 2017). 
Tabela 1 - Valores médios das características químicas e da densidade básica da madeira de Eucalyptus benthamii, E. dunnii, E. grandis e E. saligna

\begin{tabular}{|c|c|c|c|c|c|}
\hline Espécie & & $\begin{array}{c}\text { Lignina } \\
\text { Klason (\%) }\end{array}$ & $\begin{array}{c}\text { Lignina Total } \\
(\%)\end{array}$ & $\begin{array}{c}\text { Holocelulose } \\
\text { (\%) }\end{array}$ & $\begin{array}{c}\text { Densidade } \\
\text { básica } \\
\left(\mathrm{kg} / \mathrm{m}^{3}\right)\end{array}$ \\
\hline \multirow{3}{*}{ Eucalyptus benthamii } & Mín & 24,6 & 28,1 & 56,2 & 357,0 \\
\hline & Méd & 27,7 & 31,3 & 64,8 & 414,7 \\
\hline & Máx & 30,7 & 34,4 & 68,2 & 491,0 \\
\hline \multirow{5}{*}{ Eucalyptus dunnii } & $\mathrm{Cv}$ & 4,3 & 3,8 & 2,8 & 7,1 \\
\hline & Mín & 22,8 & 27,3 & 66,4 & 400,0 \\
\hline & Méd & 23,6 & 27,8 & 68,8 & 444,5 \\
\hline & Máx & 24,3 & 29,2 & 69,7 & 502,0 \\
\hline & $\mathrm{Cv}$ & 2,5 & 2,9 & 2,0 & 6,5 \\
\hline \multirow{3}{*}{ Eucalyptus grandis } & Mín & 24,9 & 29,0 & 60,0 & 349,0 \\
\hline & Méd & 28,0 & 31,6 & 62,2 & 373,2 \\
\hline & Máx & 34,3 & 36,8 & 68,4 & 415 \\
\hline \multirow{5}{*}{ Eucalyptus saligna } & $\mathrm{Cv}$ & 13,6 & 9,8 & 5,3 & 6,7 \\
\hline & Mín & 24,8 & 27,9 & 65,1 & 395,0 \\
\hline & Méd & 26,9 & 30,3 & 66,6 & 420,0 \\
\hline & Máx & 28,8 & 32,2 & 69,8 & 446,0 \\
\hline & $\mathrm{Cv}$ & 5,2 & 5,0 & 2,9 & 7,9 \\
\hline
\end{tabular}

Onde: Mín: Mínimo; Méd: Média; Máx: Máximo e CV: coeficiente de variação

Para híbridos de Eucalyptus urophylla x Eucalyptus grandis Zanuncio et al. (2013) mencionaram teor de lignina solúvel entre 2,8 e 3,1\% e Klason entre 27,5 e 30,2\%. Neiva et al. (2015) investigaram 12 espécies de Eucalyptus plantadas em Portugal, com seis anos de idade, e encontraram porcentagens de lignina solúvel na faixa de 2,2 a 3,8, lignina Klason 18,5 a 28,1 e lignina total 21,6 a 30,8 . Souza et al. (2017) determinaram para oito clones comerciais plantados na região centro oeste do Brasil teor de lignina Klason entre 22,3 e 25,8\%. A porcentagem de lignina é propensa a diminuir com a idade da árvore, pois madeiras mais jovens geralmente apresentam maior proporção de lenho juvenil, que é mais rico em lignina quando comparado ao lenho adulto (Estopa, 2017).

Sendo a remoção da lignina o principal objetivo dos processos de polpação, madeiras com baixos teores requerem condições mais brandas para atingirem determinado número Kappa. Assim, um baixo teor de lignina favorece o desempenho da atividade e proporciona economia de reagentes. Altos teores de lignina, por outro lado, obrigam o uso de condições mais severas de cozimento, degradando além da lignina os carboidratos da madeira. Analisando os critérios de qualidade da madeira de eucalipto para produção de celulose kraft, Gomide et al. (2010) afirmam que o alto teor de lignina afetou negativamente o rendimento, com uma correlação linear simples de $-0,45(p<0,05)$.

A quantidade total de polissacarídeos em Eucalyptus benthamii, mensurados como holocelulose, foi de 64,8\%, superior ao reportado por Estopa (2017) para a mesma espécie com quatro anos de idade (61,7\%). Por outro lado, o valor foi inferior ao encontrado por Alves et al. (2011) aos 6 anos de idade (68\%). Segura et al. (2017) avaliando 45 clones de Eucalyptus geneticamente melhorados, encontraram porcentagem de holocelulose de 68,7\%. Para cinco clones de Eucalyptus urograndis aos dois anos de idade, Hsing et al. (2016) verificaram altos teores de holocelulose, que variaram de 68,3 a $72,8 \%$.

O efeito da idade no teor de carboidratos na madeira ainda não é bem esclarecido. Trugilho et al. (1996) referem que em árvores de Eucalyptus saligna, avaliadas entre 12 e 48 meses de idade, houve aumento nos teores de holocelulose, porém, com tendência de estabilização entre 36 e 48 meses, quando ocorreu o início da formação da madeira adulta. Por outro lado, Neves et al. (2013) observaram redução no teor de holocelulose com o 
aumento da idade de clones de Eucalyptus spp. Santana et al. (2012) reportam em sua pesquisa que os teores de holocelulose não foram afetados pela idade e nem pela classe de diâmetro das árvores de eucalipto amostradas.

A holocelulose está relacionada ao rendimento do processo de produção de celulose. Alto conteúdo é desejável e está correlacionado positivamente com produção de polpa. Para um aproveitamento rentável na indústria de celulose da madeira de Eucalyptus benthamii, os resultados deste estudo sugerem a permanência dos indivíduos por mais de quatro anos no campo. Isto potencialmente aumentaria os teores de holocelulose e reduziria a proporção de constituintes que afetam negativamente o rendimento da atividade, tais como extrativos, cinzas e lignina.

A densidade básica das amostras variou entre $357 \mathrm{~kg} / \mathrm{cm}^{3}$ e $491 \mathrm{~kg} / \mathrm{cm}^{3}$, sendo a média de $414,7 \mathrm{~kg} / \mathrm{m}^{3}$. O valor médio encontrado é relativamente inferior aos determinados por Nisgoski et al. (1998), de $470 \mathrm{~kg} / \mathrm{m}^{3}$ e por Alves et al. (2011), de $472 \mathrm{~kg} / \mathrm{m}^{3}$, porém as amostras tinham, respectivamente, sete e seis anos de idade, superiores as analisadas neste estudo.

A densidade básica da madeira de Eucalyptus benthamii é superior ao encontrado por Souza et at. (2017) em E. camaldulensis $\left(389 \mathrm{~kg} / \mathrm{m}^{3}\right)$, também aos quatro anos de idade. Porém, mostra-se menor quando comparada às espécies comercialmente utilizadas no Brasil, com idades semelhantes, como Eucalyptus grandis (440 kg/m³ , Sette Junior et al. 2012); Eucalyptus cloeziana $\left(590 \mathrm{~kg} / \mathrm{m}^{3}\right)$ e E. urophylla $\left(460 \mathrm{~kg} / \mathrm{m}^{3}\right)$ conforme estudos de Trugilho et al. (2015), e do híbrido E. urophylla $\times$ E. grandis $\left(460 \mathrm{~kg} / \mathrm{m}^{3}\right)$ avaliado por Medeiros et al. (2016).

A densidade básica, embora nunca deva ser tomada como propriedade isolada na seleção de espécies, é um dos principais parâmetros considerados na avaliação da qualidade da madeira para a produção de polpa celulósica, fornecendo indicações bastante precisas sobre os níveis de impregnação dos cavacos, rendimento e custos do processo. Os valores considerados ideais para produção de celulose ficam em torno de $450 \mathrm{~kg} / \mathrm{m}^{3}$, com limites entre 400 e 550 kg/m (Adi et al., 2014; Clark \& Hicks, 2003; Foelkel et al., 1992; Mokfienski et al., 2008). Nesse sentido, mesmo em idade precoce de corte, Eucalyptus benthamii já demonstra potencialidade para este segmento industrial.

\section{Calibração dos modelos NIRS}

O modelo matemático de lignina Klason apresentou alta correlação, com $\mathrm{R}^{2}$ de 0,82 e RMSECV de 0,64\% (Figura 1A), com apenas duas variáveis latentes explicando a variância total dos dados. Foram necessários para calibração a exclusão de sete amostras. Os resultados demonstram o potencial de utilização deste modelo na predição de amostras desconhecidas. Altos valores de $\mathrm{R}^{2}$, como bem mencionado por Ramadevi et al. (2016), implicam em maior correlação com os valores da reta obtida, indicando maior ajuste entre os métodos laboratoriais e as análises em NIRS. 

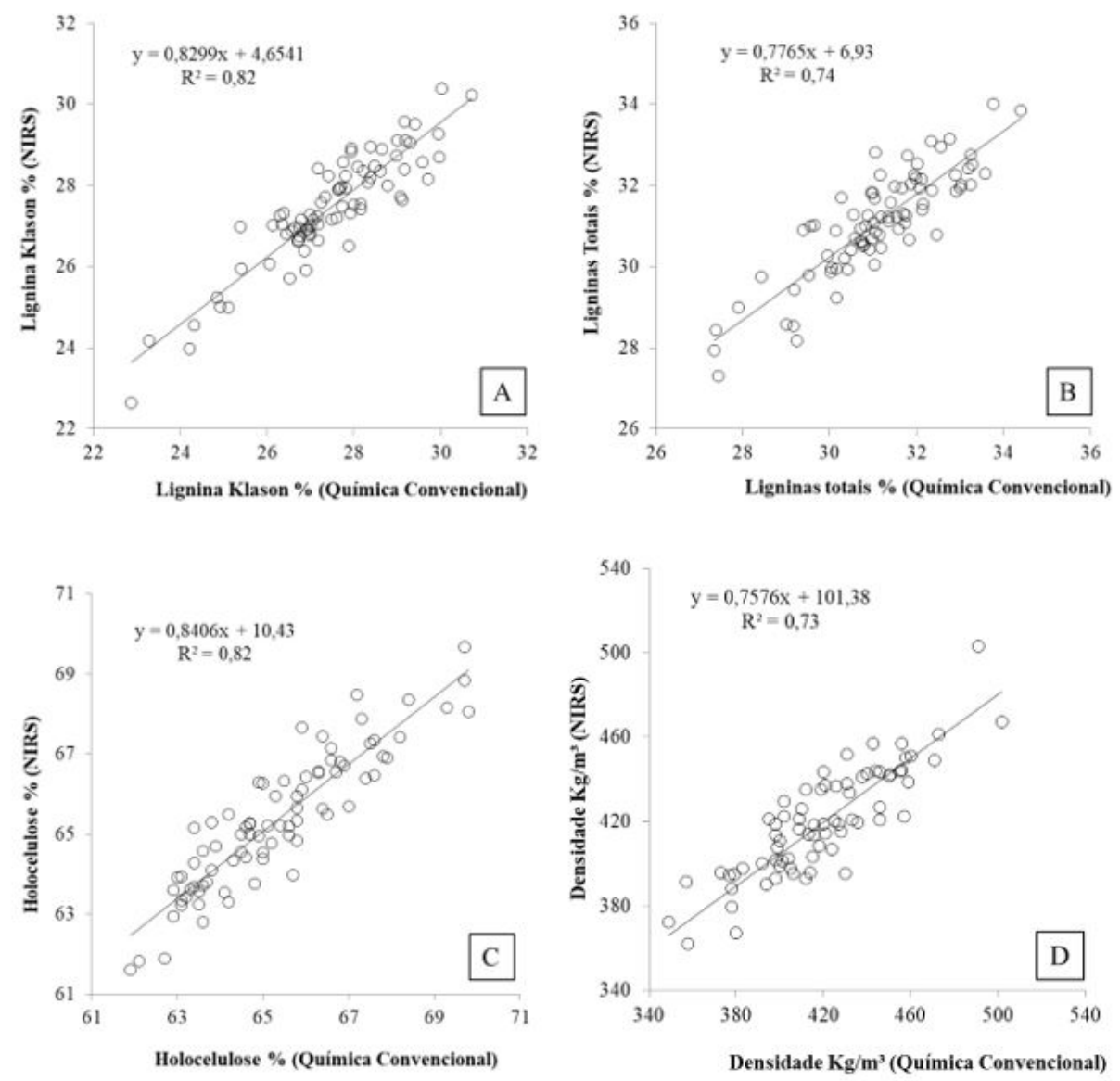

Figura 1 - Correlação entre os valores determinados convencionalmente em laboratório e os valores preditos por NIRS para lignina Klason (A) e lignina total (B) Holocelulose (C) e densidade básica (D) da madeira de Eucalyptus benthamii

Para predizer o teor de lignina Klason em Eucalyptus, Souza (2008) ajustou calibrações com coeficiente de determinação $\mathrm{R}^{2}$ de 0,75 e erro o médio da validação cruzada RMSECV de $1,00 \%$. Poke \& Raymond (2006) calibraram modelos com R $\mathrm{R}^{2}$ de 0,78 e RMSECV $1,02 \%$, ambos com capacidade de predição inferiores aos desenvolvidos neste estudo.

O modelo de calibração para a lignina total apresentou parâmetros estatísticos promissores, com $\mathrm{R}^{2}$ de 0,74 e RMSECV de 0,74\% (Figura 1B). Foram excluídas três amostras do conjunto de dados e o valor de RPD de 1,95. Resultados semelhante são frequentemente relatados na literatura, pois, entre os parâmetros de qualidade da madeira, menciona Souza (2008), o teor de lignina é um dos que apresentam melhores respostas nos modelos de predição. Poke \& Raymond (2006) observaram $\mathrm{R}^{2}=0,78$ quando o NIRS foi utilizado para estimativa do teor de lignina em madeira sólida de Eucalyptus globulus. Milagres et al. (2013) desenvolveram modelos promissores $\left(\mathrm{R}^{2}=0,71\right)$ para predizer as propriedades físicas e químicas de madeiras de Eucalyptus, com idades variando de 3 a 7 anos. Tyson et al. (2009), em seus estudos com 140 árvores do gênero Eucalyptus, encontraram valores de $R^{2}$ de 0,76, sendo considerados pelos referidos autores como satisfatórios.

A fração de holocelulose pode ser estimada na madeira por um modelo com seis variáveis latentes, seis outliers, coeficiente de determinação de 0,82 e um erro de validação cruzada de 0,76\% (Figura 1C). Estopa (2017) utilizando o mesmo número de variáveis latentes, calibrou para Eucalyptus benthamii, modelos com estatísticas inferiores $\left(R^{2}=0,69\right.$ e RMSECV $=0,61 \%)$. Hou \& Li $(2011)$ desenvolveram um modelo com alto $R^{2}(0,98)$ e baixo RMSECV $(0,34 \%)$, porém utilizando oito variáveis para explicar a variância total do conjunto de dados. Ishizuka et al. (2014) para madeiras deterioradas calibraram para o teor de lignina e holocelulose $\mathrm{R}^{2}$ de 0,97 e 0,96, respectivamente. 
Zhou et al. (2015) demonstram em seus estudos a acurácia da espectroscopia NIRS quando comparada as análises químicas convencionais da madeira. Os autores encontraram altos coeficientes de correlação na predição do teor de holocelulose de diferentes folhosas $\left(R^{2}=0,93\right)$. Igualmente, modelos com boas calibrações para holocelulose $\left(R^{2}=0,79\right.$ e RMSECV $=1,19 \%$ ) foram relatados por Zhou et al. (2016) em amostras de Eucalyptus dunnii.

O modelo de calibração para estimar a densidade básica da madeira apresentou coeficiente de determinação de 0,73 e erro de validação cruzada de $16 \mathrm{Kg} / \mathrm{m}^{3}$ (Figura 1D). Três variáveis latentes foram utilizadas para explicar a variância total do conjunto amostral, e um grande número de amostras (13 outliers) precisou ser removido do conjunto inicial de dados.

A exclusão de amostras anômalas neste estudo, verificadas pelo programa estatístico do software OPUS Quant 6.2, foi necessária para a melhoria da qualidade da calibração. Entretanto, a supressão de uma grande quantidade de amostras outliers resulta na diminuição da representatividade e eliminação de informações importantes dos dados (Pasquini, 2003).

A espectroscopia na região do infravermelho próximo tem se mostrado eficaz na determinação da densidade básica da madeira por diversos autores (Carneiro et al., 2010; Lazzarotto \& Magalhães, 2014; Magalhães et al., 2006). Modelos estatísticos com excelentes coeficientes de determinação foram encontrados por Lazzaroto et al. (2016) e Milagres et al. (2013) em estudos usando o NIRS para estimar, respectivamente, a densidade básica da madeira de Pinus $\left(R^{2}=0,94\right)$ e Eucalyptus $\left(R^{2}=0,89\right)$.

Na literatura são encontrados trabalhos utilizando NIRS para predizer a densidade básica da madeira de Eucalyptus benthamii. Lazzarotto \& Magalhães (2014) calibraram o modelo com coeficiente de determinação de predição de 0,62 e erro padrão de predição de $27 \mathrm{~kg} / \mathrm{m}^{3}$. Estopa (2017) desenvolveram baixas correlações com R $\mathrm{R}^{2}$ de 0,37 e RMSECV de $26 \mathrm{~kg} / \mathrm{m}^{3}$. Os valores reportados pelos autores são inferiores aos encontrados neste estudo, confirmando a qualidade de predição do modelo gerado.

A relação de desempenho do desvio (RPD) da lignina Klason (2,36), lignina total $(1,95)$, holocelulose $(2,39)$ e da densidade básica $(1,91)$ demonstra o potencial dos modelos para avaliar a qualidade da madeira na indústria de celulose. No campo das ciências florestais, Schimleck et al. (2003) consideram um RPD maior que 1,5 satisfatório para leituras e predições preliminares, bem como para seleção de árvores em programas de melhoramento genético. Para amostras complexas como as de madeira, podem ser usados modelos com valores de RPD acima de 1,5 para uma triagem preliminar (Prades et al., 2014; Todorovic et al., 2015).

\section{Predição das propriedades da madeira de Eucalyptus benthamii para produção de celulose}

A estimativa da lignina Klason por NIR agrupa a grande maioria dos E. benthamii (76\%) com teores entre 27 e $28,5 \%$, menos de $4 \%$ com valores considerados excelentes, abaixo de $24 \%$ e, cerca de $11 \%$ do total das amostras, com valores superiores a $30 \%$ de lignina (Figura 2A). Estes últimos, inclusive, indicam que, mesmo sendo madeira de folhosa, algumas amostras atingem teores de lignina Klason característicos de madeiras de coníferas. O valor médio predito por espectroscopia para as 1.190 árvores foi de $27 \%$, enquanto as análises de laboratório das 87 amostras apontaram 27,7\%. 


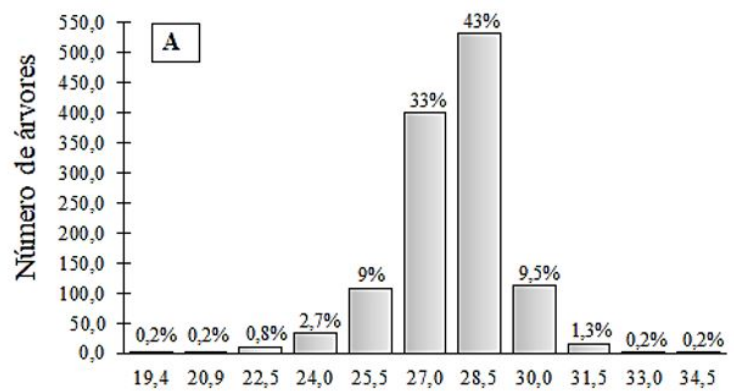

Lignina Klason (\%)

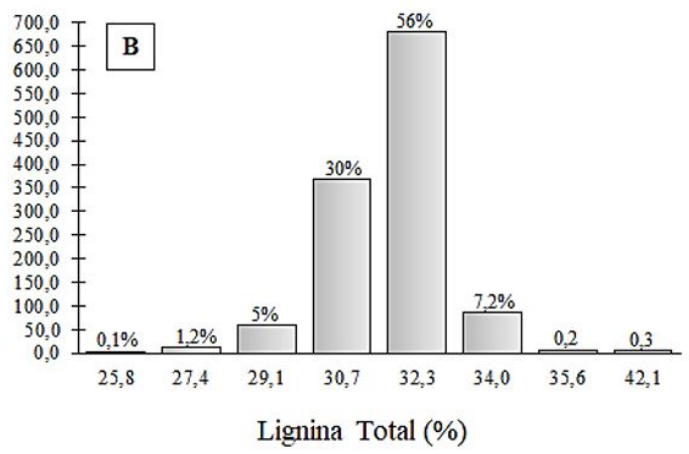

Lignina Total (\%)

Figura 2 - Distribuição dos valores preditos por espectroscopia NIRS para o teor de lignina Klason (A) e lignina total (B) para as árvores de Eucalyptus benthamii

Conforme as predições do NIR, $86 \%$ dos Eucalyptus apresentam teor de lignina total entre 30,7 e $32,3 \%$, valores considerados aceitáveis para o gênero. Os valores a esquerda do gráfico demonstram a possibilidade de seleção de árvores no povoamento com até $25,8 \%$ de lignina. A técnica de espectroscopia no infravermelho estimou o teor médio de lignina para todo povoamento de $31 \%$, e no laboratório este valor foi de 31,3\%.

A porcentagem de lignina total e Klason na madeira, aliada à sua estrutura química, afetam, diretamente, o processo de polpação. Madeiras com baixos teores desses componentes, e com a relação siringil/guaiacil elevada, são mais fáceis de deslignificarem e proporcionam maiores rendimentos finais (Gomide et al., 2010). Pelo pressuposto, tanto os teores de lignina, como a sua estrutura, devem ser considerados em uma avaliação básica da madeira, compreendendo um mínimo necessário de informações para uma caracterização preliminar de qualidade desse material.

O teor estimado de holocelulose está concentrado entre 64,8 e $67,1 \%$, com aproximadamente 923 árvores do povoamento apresentando valores neste intervalo. Embora em número reduzido, $1,6 \%$ da totalidade dos eucaliptos mostram teores de carboidratos acima de 70\% (Figura 3A). A média de holocelulose predita por NIR e em laboratório foi de, respectivamente $65,4 \%$ e $64,8 \%$.

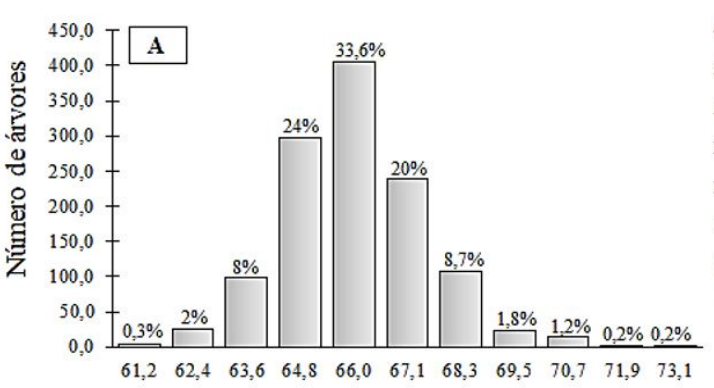

Holocelulose (\%)

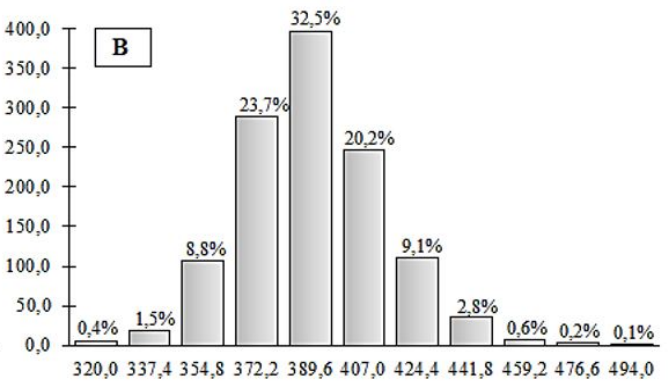

Densidade $\left(\mathrm{Kg} / \mathrm{m}^{3}\right)$

Figura 3 - Distribuição dos valores preditos por espectroscopia NIRS do teor de holocelulose (A) e densidade básica (B) para as árvores de Eucalyptus benthamii

Na seleção de árvores para produção de polpa celulósica, o percentual de holocelulose deve ser considerado como característica química fundamental, uma vez que afeta diretamente o rendimento da polpação e o potencial de produção industrial. Na prática, comenta Fantuzzi Neto (2012), a grande maioria das empresas recomendam a densidade básica, o teor de lignina e extrativos como critérios de qualidade da madeira a serem determinados para seleção de árvores para polpação, o teor de polissacarídeos é obtido como valor resposta após determinados tais parâmetros.

A estimativa da densidade básica nas espécies variou entre $320 \mathrm{~kg} / \mathrm{m}^{2}$ a $490 \mathrm{~kg} / \mathrm{m}^{3}$, com a maioria delas, $32,5 \%$, apresentando valor de $389,6 \mathrm{~kg} / \mathrm{m}^{3}$ (Figura 3B). A densidade aos quatro 
anos é relativamente menor quando comparada às espécies comercialmente utilizadas no Brasil com idades semelhantes (Eucalyptus grandis $-440,0 \mathrm{~kg} / \mathrm{m}^{3}$, E. urophylla $-460,0 \mathrm{~kg} / \mathrm{m}^{3} \mathrm{e}$ E. urograndis $-460,0 \mathrm{~kg} / \mathrm{m}^{3}$ ). Adicionalmente, outros estudos mencionam baixas densidades básicas para o Eucayptus benthamii (Nisgoski et al., 1998; Alves et al., 2011; Estopa, 2017). O mais importante neste caso, é a existência dentro do povoamento de árvores com valores ótimos, de 476,6 e $494,0 \mathrm{~kg} / \mathrm{m}^{3}$, que poderão compor um programa de melhoramento florestal, elevando a qualidade da madeira da espécie para o setor de celulose.

Cabe lembrar que os resultados obtidos pelas análises NIRS serão uteis nas decisões futuras para o melhoramento genético do povoamento de Eucayptus benthamii. Assim, é possível identificar pelas estimativas as amostras que reúnem melhores valores de holocelulose e densidade básica, e menores teores de lignina Klason e totais, por exemplo, características que refletem diretamente na qualidade da madeira para a produção de polpa celulósica.

Adicionalmente, modelos NIRS possibilitaram a avaliação precoce de características químicas e físicas importantes para o processo de produção de polpa celulósica, além da redução de custos com análises químicas, que poderão ser otimizadas.

A competitividade da indústria de polpa kraft está atrelada a possibilidade de obtenção de árvores matrizes superiores para formação de florestas, onde a matéria-prima seja homogênea e de alta qualidade. Associar característica silviculturais com às tecnológicas da madeira, torna-se então, tarefa crucial para o sucesso do setor.

\section{CONCLUSÃO}

Os modelos matemáticos de calibração mostram-se eficientes para a determinação do teor de lignina Klason e lignina total, do teor de holocelulose e da densidade básica da madeira de Eucalyptus benthamii. Os modelos também realizaram a predição destas características para as 1.190 árvores do povoamento. Resultados que recomendam sua utilização na indústria de celulose para predição de amostras desconhecidas. Desta forma, a técnica NIRS pode ser aplicada para predizer a qualidade da madeira desta espécie para a indústria de celulose de forma não-destrutiva.

\section{AGRADECIMENTOS}

Os autores agradecem a CMPC Celulose Riograndense e ao Conselho Nacional de Desenvolvimento Científico e Tecnológico - CNPQ pelo financiamento da pesquisa.

\section{REFERÊNCIAS}

Adi, D. S., Risanto, L., Damayanti, R., Rullyati, S., Dewi, L. M., Susanti, R., Dwianto, W., Hermiati, E., \& Watanabe, T. (2014). Exploration of unutilized fast growing wood species from secondary forest in central kalimantan: study on the fiber characteristic and wood density. Procedia Environmental Sciences, 20, 321-327. http://dx.doi.org/10.1016/j.proenv.2014.03.040.

Alves, A. M. M., Simões, R. F. S., Santos, C. A., Potts, B. M., Rodrigues, J., \& Schwanninger, M. (2012). Determination of Eucalyptus globulus wood extractives content by NIR-based PLS-R models: comparison between extraction procedures. Journal of Near Infrared Spectroscopy, 20(2), 275-285. http://dx.doi.org/10.1255/jnirs.987.

Alves, A., Simões, R., Stackpole, D. J., Vaillancourt, R. E., Potts, B. M., Schwanninger, M., \& Rodrigues, J. C. (2011). Determination of the syringyl/guaiacyl ratio of Eucalyptus globulus wood lignin by near infrared-based partial least squares regression models using analytical pyrolysis as the reference method. Journal of Near Infrared Spectroscopy, 19(5), 343-348. http://dx.doi.org/10.1255/jnirs.946.

Carneiro, M. E., Magalhães, W. L. E., De Muniz, G. I. B., \& Schimleck, L. R. (2010). Near infrared spectroscopy and chemometrics for predicting specific gravity and flexural modulus of elasticity of Pinus spp. veneers. Journal of Near Infrared Spectroscopy, 18(6), 481-489. http://dx.doi.org/10.1255/jnirs.911.

Clark, N. B., \& Hicks, C. C. (2003). Evaluation of the pulpwood quality of 13 lesser-known eucalypt species. Appita Journal, 52(3), 203-212. 
Estopa, R. A. (2017). Fenotipagem via nir e predição genômica em Eucalyptus benthamii (Tese de doutorado). Universidade Federal do Paraná, Curitiba.

Fantuzzi Neto, H. (2012). Qualidade da madeira de Eucalyptus spp. para produção de celulose Kraft (Tese de doutorado). Universidade Federal de Viçosa, Viçosa.

Foelkel, C., Mora, E., \& Menochelli, S. (1992). Densidade básica: sua verdadeira utilidade como índice da qualidade da madeira de eucalipto para produção de celulose. O Papel, 53(5), 35-40.

Gierlinger, N., Schwanninger, M., Hinterstoisser, B., \& Wimmer, R. (2002). Rapid determination of heartwood extractives in Larix sp. by means of Fourier transform near infrared spectroscopy. Journal of Near Infrared Spectroscopy, 10(3), 203-214. http://dx.doi.org/10.1255/jnirs.336.

Gomide, J. L., Colodette, J. L., Oliveira, R. C., \& Silva, C. M. (2005). Caracterização tecnológica, para produção de celulose, da nova geração de clones de Eucalyptus do Brasil. Revista Árvore, 29(1), 129137. http://dx.doi.org/10.1590/S0100-67622005000100014.

Gomide, J. L., Fantuzzi Neto, H., \& Regazzi, A. J. (2010). Análise de critérios de qualidade da madeira de eucalipto para produção de celulose Kraft. Revista Árvore, 34(2), 339-344. http://dx.doi.org/10.1590/S0100-67622010000200017.

Hein, P. R. G., Lima, J. T., Trugilho, P. F., \& Chaix, G. (2012). Estimativa do ângulo microfibrilar em madeira de Eucalyptus urophylla $\times$ E. grandis por meio da espectroscopia no infravermelho próximo. Floresta e Ambiente, 19(2), 194-199. http://dx.doi.org/10.4322/floram.2012.023.

Hou, S., \& Li, L. (2011). Rapid characterization of woody biomass digestibility and chemical composition using near-infrared spectroscopy. Journal of Integrative Plant Biology, 53(2), 166-175. PMid:21261813. http://dx.doi.org/10.1111/j.1744-7909.2010.01003.x.

Hsing, T. Y., Paula, N. F., \& Paula, R. C. (2016). Características dendrométricas, químicas e densidade básica da madeira de híbridos de Eucalyptus grandis × Eucalyptus urophylla. Ciência Florestal, 26(1), 273-283. http://dx.doi.org/10.5902/1980509821119.

Indústria Brasileira de Árvores - IBÁ. (2017). Relatório anual: ano base 2017. São Paulo: IBÁ.

Ishizuka, S., Yoshimi Sakai, Y., \& Tanaka-Oda, A. (2014). Quantifying lignin and holocellulose content in coniferous decayed wood using near-infrared reflectance spectroscopy. Journal of Forest Research, 19, 233-237. http://dx.doi.org/10.1007/s10310-012-0386-6.

Kelley, S. S., Rials, T. G., Groom, L. R., \& So, C. L. (2004). Use of near infrared spectroscopy to predict the mechanical properties of six softwoods. Holzforschung, 58(3), 252-260. http://dx.doi.org/10.1515/HF.2004.039.

Lazzarotto, M., \& Magalhães, W. L. E. (2014). Uso da espectroscopia no infravermelho próximo (NIR) para predição não-destrutiva de densidade básica da madeira de Eucaliptus benthammi e Eucaliptus pellita. Colombo: Embrapa Florestas.

Lazzarotto, M., Netipanyj, R. R., Magalhães, W. L. E., \& Aguiar, A. V. (2016). Espectroscopia no infravermelho próximo para estimativa da densidade básica de madeiras de Pinus. Ciência da Madeira, 7(3), 119-126. http://dx.doi.org/10.12953/2177-6830/rcm.v7n3p119-126.

Magalhães, W. L. E., Zangiski, F., Klein, C. H., \& Higa, A. R. (2006). Uso da espectroscopia no infravermelho próximo (NIR) para predição não-destrutiva de densidade básica da madeira de Pinus taeda. Colombo: Embrapa Florestas.

Medeiros, B. L. M. A., Guimarães Junior, J. B., Ribeiro, M. X., Lisboa, F. J. N., Guimarães, I., \& Protásio, T. P. (2016). Avaliação das propriedades físicas e químicas da madeira de Corymbia citriodora e Eucalyptus urophylla x Eucalyptus grandis cultivadas no Piauí. Nativa, 4(6), 403-407. http://dx.doi.org/10.14583/2318-7670.v04n06a10.

Milagres, F. R., Gomide, J. L., Magaton, A., \& Fantuzzi Neto, H. (2013). Influência da idade na geração de modelos de espectroscopia NIR, para predição de propriedades da madeira de Eucalyptus spp. Revista Árvore, 37(6), 1165-1173. http://dx.doi.org/10.1590/S0100-67622013000600018.

Mokfienski, A., Colodette, J. L., Gomide, J. L., \& Carvalho, A. M. M. L. (2008). A importância relativa da densidade da madeira e do teor de carboidratos no rendimento de polpa e na qualidade do produto. Ciência Florestal, 18(3), 401-403. http://dx.doi.org/10.5902/19805098451.

Muniz, G. I. B., Magalhaes, W. L. E., Carneiro, M. E., \& Viana, L. C. (2012). Fundamentos e estado da arte da Espectroscopia no Infravermelho Próximo no setor de base florestal. Ciência Florestal, 22(4), 865875. http://dx.doi.org/10.5902/198050987567.

Neiva, D., Fernandes, L., Araújo, S., Lourenço, A., Gominho, J., Simões, R., \& Pereira, H. (2015). Chemical composition and Kraft pulping potential of 12 eucalypt species. Industrial Crops and Products, 66, 8995. http://dx.doi.org/10.1016/j.indcrop.2014.12.016. 
Neves, T. A., Protásio, T. P., Trugilho, P. F., Valle, M. L. A., Sousa, L. C. D., \& Vieira, C. M. M. (2013). Qualidade da madeira de clones de Eucalyptus em diferentes idades para a produção de bioenergia. Revista de Ciências Agrárias, 56(2), 139-148. http://dx.doi.org/10.4322/rca.2013.022.

Nisgoski, S., Muñiz, G. I. B., \& Klock, U. (1998). Caracterização anatômica da madeira de Eucalyptus benthamii Maiden et Cambage. Ciência Florestal, 8(1), 67-76. http://dx.doi.org/10.5902/19805098352.

Pasquini, C. (2003). Near infrared spectroscopy: fundamentals, practical aspects and analytical applications. Journal of the Brazilian Chemical Society, 14(2), 198-219. http://dx.doi.org/10.1590/S0103-50532003000200006.

Pecoraro, E., Pizzo, B., Alves, A., Macchioni, N., \& Rodrigues, J. C. (2015). Measuring the chemical composition of waterlogged decayed wood by near infrared spectroscopy. Microchemical Journal, 122, 176-188. http://dx.doi.org/10.1016/j.microc.2015.05.005.

Poke, F. S., \& Raymond, C. A. (2006). Predicting extractives, lignin, and cellulose contents using near infrared spectroscopy on solid wood in Eucalyptus globulus. Journal of Wood Chemistry and Technology, 26(2), 187-199. http://dx.doi.org/10.1080/02773810600732708.

Prades, C., Gomez-Sanchez, I., Garcia-Olmo, J., Gonzalez-Hernandez, F., \& Gonzalez-Adrados, J. R. (2014). Application of VIS/NIR spectroscopy for estimating chemical, physical and mechanical properties of cork stoppers. Wood Science and Technology, 48(4), 811-830. http://dx.doi.org/10.1007/s00226-0140642-3.

Ramadevi, P., Hegde, D. V., Varghese, M., Kamalakannan, R., Ganapathy, S. P., \& Gurumurthy, D. S. (2016). Evaluation of lignin syringyl/guaiacyl ratio in Eucalyptus camaldulensis across three diverse sites based on near infrared spectroscopic calibration modelling with five Eucalyptus species and its impact on Kraft pulp yield. Journal of Near Infrared Spectroscopy, 24(6), 529-536. http://dx.doi.org/10.1255/jnirs.1251.

Raymond, C. A., \& Muneri, A. (2001). Nondestructive sampling of Eucalytpus globulus and E. nitens for wood properties. I. Basic density. Wood Science and Technology, 35(1), 27-39. http://dx.doi.org/10.1007/s002260000078.

Rosso, S., Muniz, G. I. B., Matos, J. L. M., Haselein, C. R., Hein, P. R. G., \& Lopes, M. C. (2013). Density of Eucalyptus grandis W. Hill Ex Maiden using near infrared spectroscopy. Cerne, 19(4), 647-652. http://dx.doi.org/10.1590/S0104-77602013000400015.

Santana, W. M. S., Calegario, N., Arantes, M. D. C., \& Trugilho, P. F. (2012). Effect of age and diameter class on the propperties of wood from clonal Eucalyptus. Cerne, 18(1), 1-8. http://dx.doi.org/10.1590/S0104-77602012000100001.

Scandinavian Pulp, Paper and Board Testing Committee - SCAN. (1995). SCAN-CM 43:95: wood chips for pulp production, basic density. Stockholm: SCAN.

Schimleck, L. R., Doran, J. C., \& Rimbawanto, A. (2003). Near infrared spectroscopy for cost-effective screening of foliar oil characteristics in a Melaleuca cajuputi breeding population. Journal of Agricultural and Food Chemistry, Easton, 51(9), 2433-2437. PMid:12696917. http://dx.doi.org/10.1021/jf020981u.

Segura, T. E. S. (2015). Avaliação das madeiras de Corymbia citriodora, Corymbia torelliana e seus híbridos visando à produção de celulose Kraft branqueada (Tese de doutorado). Escola Superior de Agricultura "Luiz de Queiroz", Universidade de São Paulo, Piracicaba.

Segura, T. E. S., Pimenta, L. R., Mattiazzo, F. B., Silva, F. M., Cruz, J. A., \& Souza, L. O. (2017). Programa de controle da qualidade da madeira na Eldorado Brasil celulose. O Papel, 78(7), 82-89.

Sette Junior, C. R., Oliveira, I. R., Tomazello Filho, M., Yamaji, F. M., \& Laclau, J. P. (2012). Efeito da idade e posição de amostragem na densidade e características anatômicas da madeira de Eucalyptus grandis. Revista Árvore,, 36(6), 1183-1190. http://dx.doi.org/10.1590/S0100-67622012000600019.

Souza, F. M. L., Pupo, C. H., Sereghetti, G. C., Sansígolo, C. A., Ferreira, J. P., Silva, R. B., \& Garcia, D. P. (2017). Características de crescimento, densidade básica e composição química da madeira de Eucalyptus spp na região de Ribas do Rio Pardo-MS. Brazilian Journal of Biosystems Engineering, 11(4), 350-359. http://dx.doi.org/10.18011/bioeng2017v11n4p350-359.

Souza, L. C. (2008). Espectroscopia na região do infravermelho próximo para predição de características da madeira para produção de celulose (Tese de doutorado). Universidade Federal de Viçosa, Viçosa.

Technical Association of the Pulp and Paper Industry - TAPPI. (1991). TAPPI T UM 250: acid-soluble lignin in wood and pulp. Atlanta: TAPPI Press.

Technical Association of the Pulp and Paper Industry - TAPPI. (1999). TAPPI T203 CM-99: alpha-, beta- and gamma-cellulose in pulp. Atlanta: TAPPI Press. 
Technical Association of the Pulp and Paper Industry - TAPPI. (2002). TAPPI 211 OM-02: ash in wood, pulp, paper and paperboard: combustion at $525^{\circ} \mathrm{C}$. Atlanta: TAPPI Press.

Todorovic, N., Popovic, Z., \& Milic, G. (2015). Estimation of quality of thermally modified beech wood with red heartwood by FT-NIR spectroscopy. Wood Science and Technology, 49(3), 527-549. http://dx.doi.org/10.1007/s00226-015-0710-3.

Trugilho, P. F., Goulart, S. L., Assis, C. O., Couto, F. B. S., Alves, I. C. N., Protásio, T. P., \& Napoli, A. (2015). Características de crescimento, composição química, física e estimativa de massa seca de madeira em clones e espécies de Eucalyptus jovens. Ciência Rural, 45(4), 661-666. http://dx.doi.org/10.1590/0103-8478cr20130625.

Trugilho, P. F., Lima, J. T., \& Mendes, L. M. (1996). Influência da idade nas características físicoquímicas e anatômicas da madeira de Eucalyptus saligna. Cerne, 2(1), 94-116.

Tyson, J. A., Schimleck, L. R., Aguiar, A. M., Abad Muro, J. I., \& Rezende, G. D. S. P. (2009). Adjusting near infrared wood property calibrations for central Brazil to predict the wood properties of samples from Southern Brazil. Journal Appita, 62(1), 46-51.

Zanuncio, A. J. V., Colodette, J. L., Gomes, F. J. B., Carneiro, A. C. O., \& Vital, B. R. (2013). Composição química da madeira de eucalipto com diferentes níveis de desbaste. Ciência Florestal, 23(4), 755-760. http://dx.doi.org/10.5902/1980509812359.

Zhou, C., Jiang, W., Via, B. K., Chetty, P. M., \& Swain, T. (2016). Monitoring the chemistry and monosaccharide ratio of Eucalyptus dunnii wood by near infrared spectroscopy. Journal of Near Infrared Spectroscopy, 24(6), 537-548. http://dx.doi.org/10.1255/jnirs.1254.

Zhou, C., Jiang, W., Via, B. K., Fasina, O., \& Han, G. (2015). Prediction of mixed hardwood lignin and carbohydrate content using ATR-FTIR and FT-NIR. Carbohydrate Polymers, 121, 336-341. PMid:25659707. http://dx.doi.org/10.1016/j.carbpol.2014.11.062.

Contribuição dos autores: TB: conceituação, curadoria de dados, supervisão, validação, visualização, escrita primeira redação, MT: escrita - revisão e edição; AGS: curadoria de dados; escrita - revisão e edição;

JNCM: obtenção de financiamento; GAS: obtenção de financiamento, conceituação, administração do projeto, OPS: recursos, software, BMRTV: recursos, software. 